\title{
An autophagy-related long non-coding RNA signature for patients with colorectal cancer
}

\section{DONGYAN ZHAO ${ }^{1}$, XIZHEN SUN ${ }^{1}$, SIDAN LONG ${ }^{2}$ and SHUKUN YAO ${ }^{1,3 *}$}

\author{
${ }^{1}$ Graduate School, Peking Union Medical College and Chinese Academy of Medical Sciences, Beijing \\ 100730, China \\ ${ }^{2}$ Beijing University of Chinese Medicine, Beijing 100029, China \\ ${ }^{3}$ Department of Gastroenterology, China-Japan Friendship Hospital, Beijing 100029, China
}

Received: August 19, $2020 \bullet$ Accepted: January 29, 2021

Published online: July 5, 2021

(C) 2021 Akadémiai Kiadó, Budapest

\begin{abstract}
Aim: Long non-coding RNAs (lncRNAs) have been identified to regulate cancers by controlling the process of autophagy and by mediating the post-transcriptional and transcriptional regulation of autophagy-related genes. This study aimed to investigate the potential prognostic role of autophagy-associated lncRNAs in colorectal cancer (CRC) patients. Methods: LncRNA expression profiles and the corresponding clinical information of CRC patients were collected from The Cancer Genome Atlas (TCGA) database. Based on the TCGA dataset, autophagy-related lncRNAs were identified by Pearson correlation test. Univariate Cox regression analysis and the least absolute shrinkage and selection operator analysis (LASSO) Cox regression model were performed to construct the prognostic gene signature. Gene set enrichment analysis (GSEA) was used to further clarify the underlying molecular mechanisms. Results: We obtained 210 autophagyrelated genes from the whole dataset and found $1187 \operatorname{lncRNAs}$ that were correlated with the autophagyrelated genes. Using Univariate and LASSO Cox regression analyses, eight lncRNAs were screened to establish an eight-lncRNA signature, based on which patients were divided into the low-risk and high-risk group. Patients' overall survival was found to be significantly worse in the high-risk group compared to that in the low-risk group (log-rank $\mathrm{p}=2.731 \mathrm{E}-06)$. ROC analysis showed that this signature had better prognostic accuracy than TNM stage, as indicated by the area under the curve. Furthermore, GSEA demonstrated that this signature was involved in many cancer-related pathways, including TGF- $\beta$, p53, mTOR and WNT signaling pathway. Conclusions: Our study constructed a novel signature from eight autophagy-related lncRNAs to predict the overall survival of CRC, which could assistant clinicians in making individualized treatment.
\end{abstract}

\footnotetext{
* Corresponding author. Department of Gastroenterology, China-Japan Friendship Hospital, ${ }^{\text {nd }}$ Yinghua East Road, Chaoyang District, Beijing 100029, China. Tel./fax: +86 10 84205108. E-mail: shukunyao@126.com
} 


\section{KEYWORDS}

autophagy, long non-coding RNA, prognostic signature, colorectal cancer, TCGA

\section{INTRODUCTION}

Colorectal cancer (CRC) is one of the most common gastrointestinal malignant tumors, as well as the leading cause of death owing to its high morbidity and mortality rates [1]. The global burden of CRC is expected to increase in developing countries and reach more than 2.2 million new cases and 1.1 million deaths by 2030 [2]. Although multiple treatments, such as chemotherapy, surgery, targeted therapy, radiotherapy, and immunotherapy, have been shown to reduce relapse and improve the survival of CRC patients, the overall five-year survival rate for patients suffering from advanced CRC remains very poor [1,3]. Unexpectedly, patients diagnosed with early-stage CRC with an early operation (early surgery) still have a $20-30 \%$ recurrence rate and are likely to progress into a higher histological grade of malignancy [4]. To date, patient prognosis still heavily relies on the American Joint Committee on Cancer (AJCC) TNM staging system. However, it is still a challenge to predict the survival of patients with CRC by using the current prognostic system because of the wide variability of outcomes and genetic heterogeneity among patients [5-7]. Thus, identifying reliable prognostic biomarkers to promote our understanding of cancer development and better predict the overall survival and prognosis of CRC is greatly desirable.

Autophagy, a protein degradation system, is a critical and highly conserved regulatory process for cells to maintain function normally by lysosomal degradation of unnecessary or damaged components [8]. It is a system that runs at basal level in all cells, but the rate of autophagy increases during times of inflammation, hypoxia, stress, or low nutrition to provide an additional energy source for the cell [9]. Paradoxically, although autophagy principally plays an adaptive role to protect cells against various pathologies, the process of autophagy can also promote carcinogenesis by facilitating the survival of tumor cells under pathological microenvironmental stress conditions [10]. Of note, growing evidence has demonstrated the important role of autophagy in the progression process of CRC [11]. Data from Niklaus et al. indicated that intact activated autophagy is correlated with a poor prognosis in colon cancer [12]. The autophagy gene Atg4B has been confirmed as an oncogene that facilitates tumor proliferation and a negative regulator of autophagy in CRC cells [13]. Recently, some studies have tried to find new effective targeted treatments by studying the function of autophagy [14-16]. For instance, niclosamide, a new drug for treating metastatic CRC, has been confirmed to inhibit WNT/ $\beta$ catenin signaling in CRC cells by degrading components of the pathway via autophagy [17]. Further study of autophagy and its prevalence in patients with CRC will uncover its potential therapeutic use.

With the development of sequencing technology, the transcriptomes of many organisms have been surveyed, and these studies identified thousands of transcripts longer than 200 nucleotides in length that do not have significant protein-coding abilities and are thus termed long non-coding RNA (lncRNA) [18]. A growing amount of evidence has shown that lncRNAs regulate most cancers by controlling the process of autophagy and by modulating the post-transcriptional and transcriptional regulation of autophagy-related genes $[19,20]$. 
For CRC, abnormal expression of several lncRNAs has been proven to be markedly correlated with unfavorable prognosis. According to a recent study, increased levels of POU3F3, a long intergenic non-coding RNA, were associated with an increased risk of CRC and could promote metastasis of the neoplasm. Knockdown of POU3F3 was associated with autophagy induction in CRC cells through BMP signaling, resulting in excessive apoptosis and reduced invasion and migration capacities of CRC cells [20]. Zheng et al. demonstrated that increased HAGLROS levels were significantly associated with decreased survival times in patients with CRC and that downregulated expression of HAGLROS inhibited autophagy in CRC cells by sponging miR-100 to target and regulate the expression of autophagy gene Atg5 [21]. Therefore, abnormal expression of autophagy-related lncRNAs may have prognostic value in patients with CRC and could be exploited as a potential molecular target for cancer therapy.

Currently, gene-based prognostic signatures that incorporate other clinical parameters to predict overall survival of CRC patients have been thoroughly investigated, but no prognostic model of CRC based on autophagy-related lncRNAs has been developed. Constructing a predictive autophagy-related lncRNA signature may be a valuable strategy for predicting the prognosis of CRC patients. Thus, the objective of this study was to generate an autophagyrelated lncRNA signature in CRC patients and to further advance the personalization of CRC treatment.

\section{MATERIALS AND METHODS}

\section{Data collection}

All the Fragments per Kilobase Million (FPKM) normalized expression profile data and the corresponding clinical information for CRC patients were downloaded and collected from The Cancer Genome Atlas (TCGA) database, including 647 tumor tissues and 51 non-tumor tissues (dated to March 18, 2020, https://portal.gdc.cancer.gov/repository). TCGA database is freely available to the public, thus there was no requirement for additional ethical approval. Based on the requirement for data integration, we only included CRC patients whose records contained complete information on clinicopathological variables, including age, gender, tumor stage, survival status and survival time and those having a survival time of more than 30 days (implying a probable death caused by CRC). Hence, we included 505 CRC patients for further analysis.

\section{LncRNA profile mining}

The RNA expression data of mRNAs and lncRNAs were obtained from the TCGA dataset and the expression profiles of mRNAs were normalized by using log2 transformation. LncRNAs were identified as protein-coding genes or non-coding genes based on their Ensembl IDs, and only the long non-coding genes in NetAffx Annotation files were retained. Next, we obtained a latest list of autophagy-related genes by accessing the Human Autophagy Database (HADb, http://autophagy.lu/clustering/index.html). The correlation between the lncRNAs and autophagy-related genes was conducted by measuring Pearson correlation coefficients. Autophagy-related lncRNAs were identified with a threshold value 
of $P<0.05$ and correlation coefficient $\left|R_{2}\right|>0.3$. The 505 CRC patients were then randomly assigned to the training set $(50 \%)$ and the testing set $(50 \%)$ (as shown in Supplementary Table S1). Then we used the training set to construct the prognostic autophagy-related lncRNAs signature.

\section{Construction and confirmation of an autophagy-related IncRNA signature}

To investigate the role of survival-associated lncRNAs as predictive and prognosis markers in CRC patients, univariate Cox proportional hazards regression was performed to evaluate the associations between the expression of autophagy-related lncRNAs and the patients' overall survival by using the survival R package. Only those lncRNAs of the test set with a $P$ value less than 0.01 were selected for stepwise model selection by least absolute shrinkage and selection operator (LASSO) regression analysis, which was further performed to reduce the variable numbers and effectively avoid overfitting. The LASSO method was utilized by the package "glmnet" in the R (version 3.6.1) software. Subsequently, we constructed a prognostic predictive model using a multivariate Cox hazard model analysis and calculated the individualized risk score using the following formula: risk score $=\sum \beta \mathrm{i} \times \operatorname{expRNAi}$, where expRNA indicates the expression level of a lncRNA and $\beta$ is the regression coefficient for the corresponding lncRNA obtained from the LASSO regression model.

The testing set and the whole set were used to validate the robustness and prognostic value of this signature. According to the lncRNA-based risk score formula, patients from the training and testing groups were classified into high-risk and low-risk cohorts using the median risk score as the cutoff value. Kaplan-Meier curves and log-rank tests were utilized to compare the two groups' survival event. Receiver operating characteristic (ROC) analysis was performed to evaluate the robustness of the prognostic signature for overall survival by calculating the area under curve (AUC) in the R package of "survival ROC".

\section{Independent prognostic role of the IncRNA signature}

In order to determine whether the prognostic value of the lncRNA signature was independent of other clinicopathological parameters including age, gender, stage, and TNM status, univariate and multivariate analyses were performed to measure statistical significance. $P<0.05$ was considered statistically significant.

\section{Gene set enrichment analysis}

Gene set enrichment analysis (GSEA, http://software.broadinstitute.org/gsea/index.jsp) is a powerful computational algorithm based on the JAVA8 platform. In order to identify the enriched terms and uncover the potential mechanisms underlying the prognostic lncRNAs signature in our study, Kyoto Encyclopedia of Genes and Genomes (KEGG) functional enrichment analyses were performed by using the level of risk score as phenotype in GSEA software. Genes were ranked with the metric of absolute "signal-to-noise" value, and 1000 random sample permutations were carried out. Only KEGG pathways with nominal $P$ values $<0.05$, false discovery rates (FDR) $<25 \%$ and normalized enrichment scores (NES) $>1$ were considered to be statistically significant [22]. 


\section{Statistical analysis}

The autophagy-lncRNA interaction network was analyzed and visualized using Cytoscape software (version 3.7.2; The Cytoscape Consortium, San Diego, CA, USA). Statistical analyses were performed using R software (version 3.6.1; The R Foundation for Statistical Computing, Vienna, Austria). All GSEA analyses presented in our study were performed using a java implementation of GSEA software (version 4.0.3). A $P$-value of less than 0.05 was considered to be statistically significant unless otherwise mentioned.

\section{RESULTS}

\section{Construction of autophagy-IncRNAs coexpression networks}

A total of $14,142 \operatorname{lncRNAs}$ were obtained from the whole dataset, and the expression profiles were identified from the microarray dataset by lncRNA expression profile mining. A total of 210 autophagy-related genes were identified from the Human Autophagy Database. A coexpression network for autophagy-related genes and lncRNAs was constructed and used to find autophagyrelated $\operatorname{lncRNAs}$ with a threshold of $\left|\mathrm{R}_{2}\right|>0.3$ and $P<0.05$. Finally, we selected 1,187 autophagyrelated lncRNAs for further analysis. The correlations between the lncRNAs and the autophagyrelated genes are presented in Supplementary Table S2.

\section{Identification of an eight-IncRNA signature in patients with CRC}

To extract autophagy-related lncRNAs that actively participated in the onset and progression of CRC, all autophagy-related lncRNAs in the training dataset were subjected to univariate Cox proportional hazard regression analysis, and a total of 27 survival-associated lncRNAs were input into LASSO regression $(P<0.01$, Fig. 1A). Then, 19 key lncRNAs were selected for multivariate Cox regression analysis (Fig. 1B), and eight lncRNAs (AC027307.2, MIR210HG, FAM160A1-DT, FGD5-AS1, AC023157.3, CD27-AS1, LINC01063, AC145098.1) were further selected. We further evaluated the autophagy-lncRNA coexpression networks based on the eight genes (Supplementary Fig. S1). Multivariate Cox hazard model analysis of the association of these eight autophagy-related lncRNAs with overall survival showed that two lncRNAs were independent factors associated with good overall survival in CRC patients (FAM160A1-DT, FGD5-AS1), whereas six lncRNAs were identified to be unfavorable prognostic factors for CRC (AC027307.2, MIR210HG, AC023157.3, CD27-AS1, LINC01063, AC145098.1). The detailed results from the multivariate Cox regression analysis are presented in Table 1.

\section{Establishment and estimation of the eight-IncRNA prognostic signature}

Based on the LASSO regression model, the coefficient values were extracted and a predictive model containing eight lncRNAs was generated to calculate the risk score of every patient with the following formula: Risk score $=$ expression level of AC027307.2*0.227 + MIR210HG* 0.140 + FAM160A1-DT* $(-0.573)+$ FGD5-AS1 ${ }^{*}(-0.045)+$ AC023157.3*0.484 + CD27-AS1*0.090 + LINC01063*0.674 + AC145098.1*0.485. With the median prognostic score of the training dataset, 0.857 as the cutoff point, the CRC patients were classified into the low-risk group and 


$\begin{array}{rll}\text { A } & \text { pvalue } & \text { Harzard ratio } \\ \text { AC106820.3 } & 0.008 & 1.75(1.157-2.693) \\ \text { KMT2E-AS1 } & 0.008 & 1.101(1.026-1.182) \\ \text { AC011462.4 } & 0.008 & 1.549(1.123-2.136) \\ \text { MIR155HG } & 0.008 & 1.547(1.123-2.131) \\ \text { FAM160A1-DT } & 0.008 & 0.479(0.279-0.822) \\ \text { LINC02038 } & 0.007 & 0.510(0.311-0.835) \\ \text { AC106782.6 } & 0.007 & 1.410(1.096-1.813) \\ \text { LINC00941 } & 0.007 & 1.291(1.072-1.554) \\ \text { LINC01063 } & 0.006 & 1.618(1.147-2.283) \\ \text { AC023157.3 } & 0.006 & 1.574(1.138-2.176) \\ \text { ATP2A1-AS1 } & 0.006 & 1.227(1.061-1.420) \\ \text { ITGB2-AS1 } & 0.005 & 2.002(1.227-3.267) \\ \text { AC145098.1 } & 0.005 & 1.824(1.201-2.740) \\ \text { CD27-AS1 } & 0.004 & 1.125(1.039-1.219) \\ \text { HLA-DQB1-AS1 } & 0.003 & 1.423(1.120-1.757) \\ \text { AC156455.1 } & 0.003 & 1.260(1.080-1.470) \\ \text { AP006621.3 } & 0.003 & 1.301(1.093-1.548) \\ \text { MIRLETBHG } & 0.002 & 1.345(1.116-1.622) \\ \text { AC004687.1 } & 0.001 & 1.098(1.036-1.163) \\ \text { FGD5-AS1 } & <0.001 & 0.944(0.912-0.976) \\ \text { AP005899.1 } & <0.001 & 2.280(1.444-3.600) \\ \text { AL109615.3 } & <0.001 & 1.090(1.040-1.143) \\ \text { AC027307.2 } & <0.001 & 1.218(1.093-1.357) \\ \text { AC027796.4 } & <0.001 & 1.562(1.239-1.968) \\ \text { MIR210HG } & <0.001 & 1.215(1.100-1.343) \\ \text { AP006284.1 } & <0.001 & 1.931(1.386-2.689) \\ \text { AC010973.2 } & <0.001 & 1.960(1.440-2.668) \\ & & \end{array}$

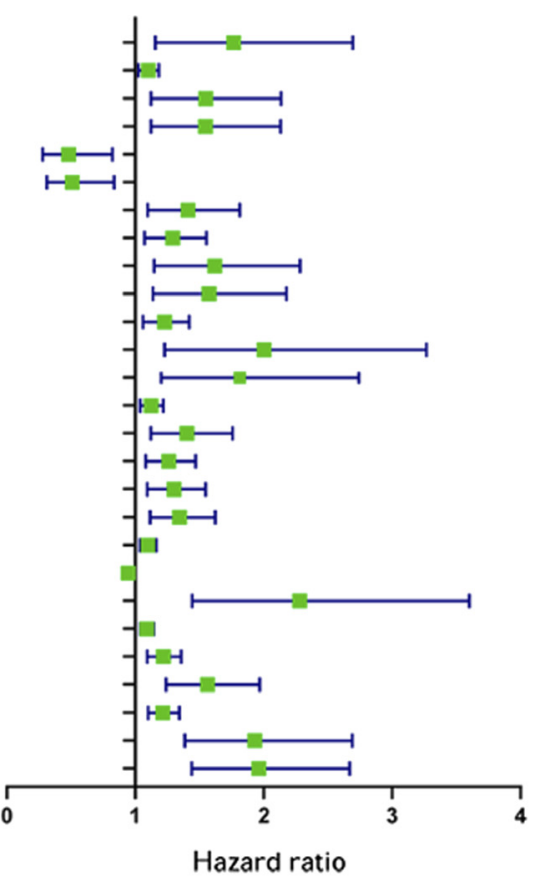

B $\begin{array}{lllllllllllllll}26 & 26 & 25 & 25 & 25 & 26 & 25 & 23 & 23 & 20 & 20 & 19 & 9 & 4 & 0\end{array}$

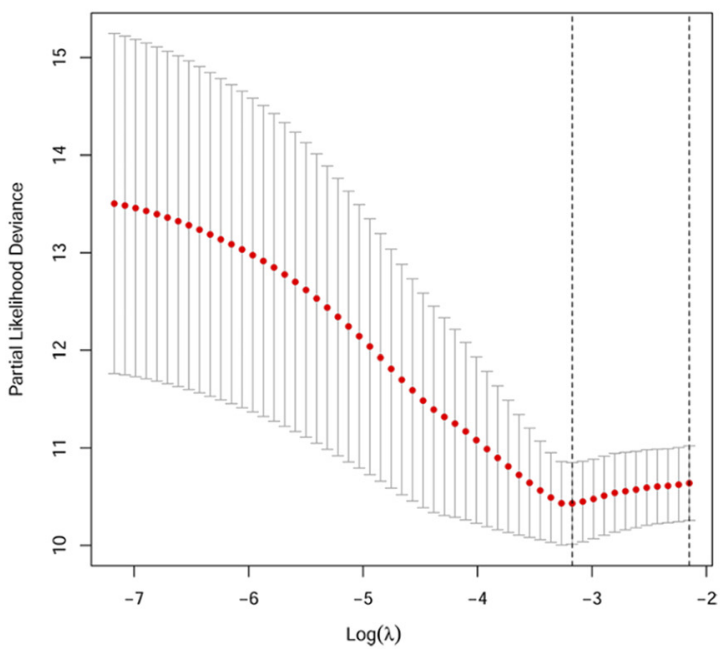

Fig. 1. Univariate Cox regression and LASSO analysis for screening prognostic features.

(A) The forest plot of 27 survival-associated lncRNAs screened out by univariate Cox proportional hazards regression. (B) The partial likelihood deviance plot presented the minimum number, which corresponds to the covariates used for multivariate Cox analysis. LASSO, least absolute shrinkage and selection operator; lncRNAs: long non-coding RNAs 
Table 1. Detailed information for 8 autophagy-related lncRNAs significantly associated with overall survival in CRC

\begin{tabular}{lcccccl}
\hline LncRNA & Ensemble ID & Coefficient & HR & HR.95L & HR.95H & $P$-value \\
\hline AC027307.2 & ENSG00000267317 & 0.227 & 1.255 & 1.126 & 1.400 & $4.24 \mathrm{E}-05$ \\
MIR210HG & ENSG00000282810 & 0.140 & 1.151 & 1.039 & 1.274 & 0.007 \\
FAM160A1-DT & ENSG00000251611 & -0.573 & 0.564 & 0.321 & 0.991 & 0.047 \\
FGD5-AS1 & ENSG00000225733 & -0.045 & 0.956 & 0.921 & 0.992 & 0.018 \\
AC023157.3 & ENSG00000276900 & 0.484 & 1.623 & 1.132 & 2.328 & 0.008 \\
CD27-AS1 & ENSG00000215039 & 0.090 & 1.094 & 1.002 & 1.195 & 0.046 \\
LINC01063 & ENSG00000232065 & 0.674 & 1.961 & 1.402 & 2.744 & $8.39 \mathrm{E}-05$ \\
AC145098.1 & ENSG00000248996 & 0.485 & 1.624 & 1.0390 & 2.540 & 0.033 \\
\hline
\end{tabular}

Abbreviations: lncRNAs: long non-coding RNAs; HR: hazard ratio; CI: confidence interval.

the high-risk group; consequently, a prognostic signature based on eight autophagy-related lncRNAs was developed.

The distribution of the risk score along with the survival status of CRC patients and the heatmap of the eight autophagy-related lncRNAs in the training dataset were plotted and are shown in Fig. 2A $(\mathrm{a}-\mathrm{c})$, which demonstrates that patients with low risk scores had a lower death rate and a longer overall survival time than those with high risk scores. Consistent with this result, the Kaplan-Meier survival curves of the low-risk and high-risk groups in the training dataset were significantly different, showing overall survival of $33.70 \%(95 \% \mathrm{CI}=19.10 \%-59.30 \%)$ and $86.80 \%(95 \% \mathrm{CI}=77.90 \%-96.70 \%)$ at five years for patients with high and low risk scores, respectively (log-rank $\mathrm{p}=2.731 \mathrm{E}-06$, Fig. $2 \mathrm{~A}(\mathrm{~d})$ ). In addition, the prognostic power of the eight-lncRNA signature in the training group was evaluated by calculating the AUC of the ROC curve. ROC analysis showed that the signature $(\mathrm{AUC}=0.817$ ) had better prognostic accuracy than TNM staging (AUC $=0.710$, Fig. 2A (e)).

\section{Performance validation of the eight-IncRNA prognostic signature}

To confirm the survival prediction power of the signature, we used the testing set $(n=252)$ and the whole set $(n=505)$ to assess the findings from the training set. The results are presented in Fig. 2B and $\mathrm{C}$ with risk score analysis, Kaplan-Meier curves and ROC curves. Consistent with the above findings, the Kaplan-Meier analysis of the two sets showed that the high-risk group had shorter overall survival time than the low-risk group (log-rank $\mathrm{p}=$ $5.578 \mathrm{E}-04$ in the testing set and log-rank $\mathrm{p}=3.07 \mathrm{E}-08$ in the whole set). The five-year survival rate was $47.50 \%(95 \% \mathrm{CI}=37.40-60.30 \%)$ for patients with high risk scores and $86.20 \%(95 \% \mathrm{CI}=79.60-93.40 \%)$ for patients with low risk scores in the whole set. In addition, the AUCs were 0.698 and 0.762 for testing set and whole set, respectively, indicating the improved performance of the eight-lncRNA signature model in predicting CRC patient survival risk.

To further explore the predictive value of the eight-lncRNA signature, Cox regression analyses were utilized based on clinicopathological parameters, including age, gender, and TNM stage (Fig. 3). Univariate and multivariate Cox regression analysis indicated that age, AJCC T 
A
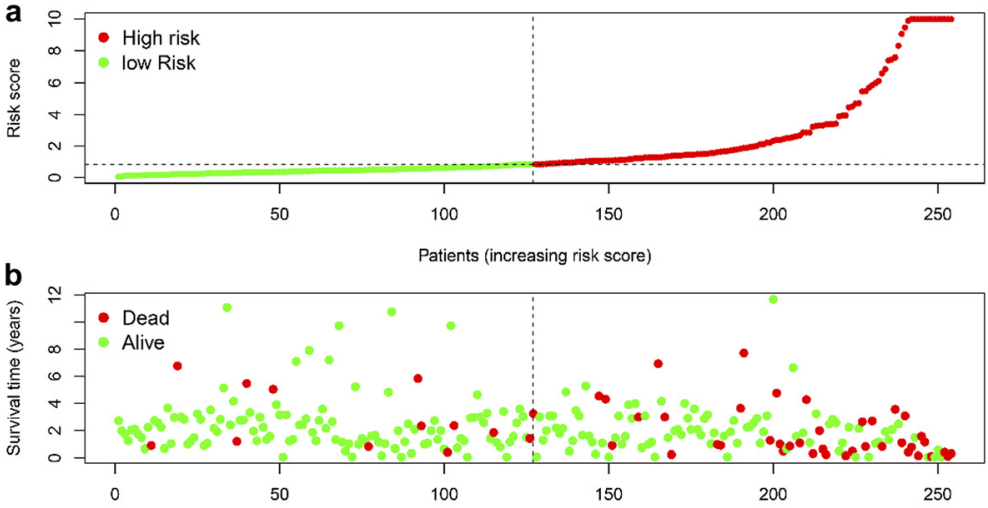

Patients (increasing risk score)

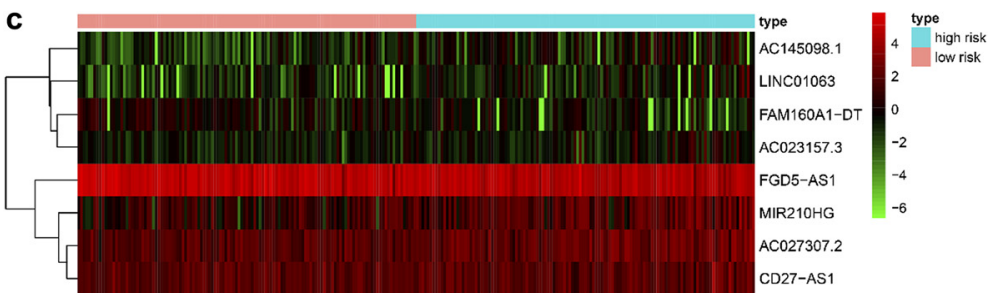

d

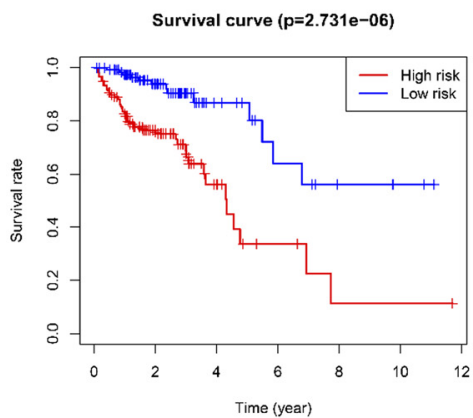

e

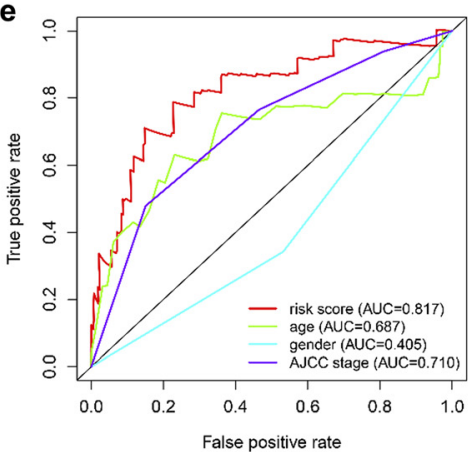

Fig. 2. Risk score analysis, Kaplan-Meier survival curves and ROC curves for the autophagy-related lncRNA signature in CRC.

(A) The distributions of the risk score (a), survival status (b) and expression profiles (c), Kaplan-Meier curve (d) and ROC curve (e) of the eight-lncRNA prognostic signature in the training set of TCGA cohort. (B) The distributions of the risk score (a), survival status (b) and expression profiles (c), Kaplan-Meier curve (d) and ROC curve (e) of the eight-lncRNA prognostic signature in the testing set of TCGA cohort. (C) The distributions of the risk score (a), survival status (b) and expression profiles (c), Kaplan-Meier curve (d) and ROC curve (e) of the eight-lncRNA prognostic signature in the whole set of TCGA cohort. CRC: colorectal cancer; lncRNAs: long non-coding RNAs; ROC: receiver operating characteristic; TCGA: The Cancer Genome Atlas 


\section{B}

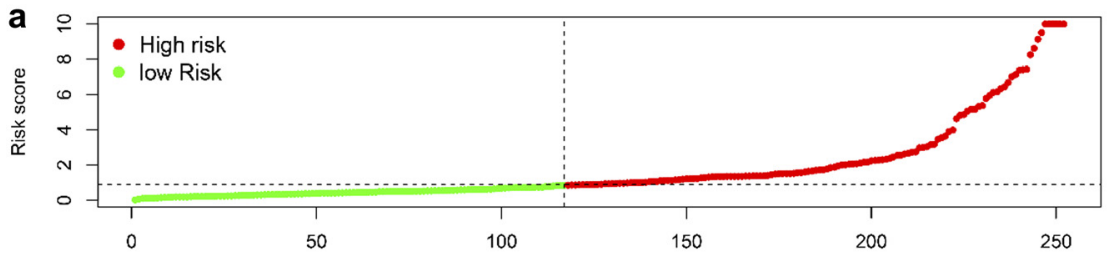

b

Patients (increasing risk score)

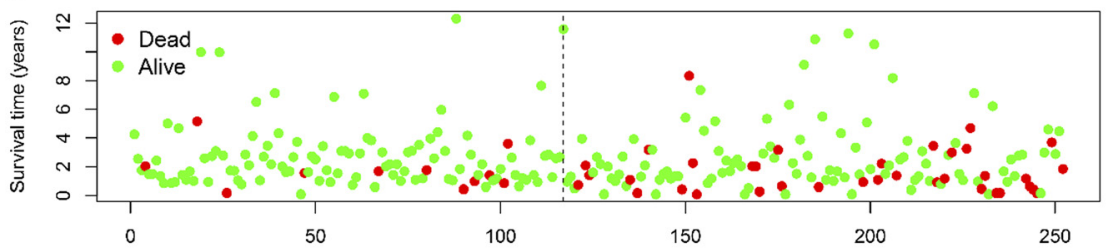

Patients (increasing risk score)

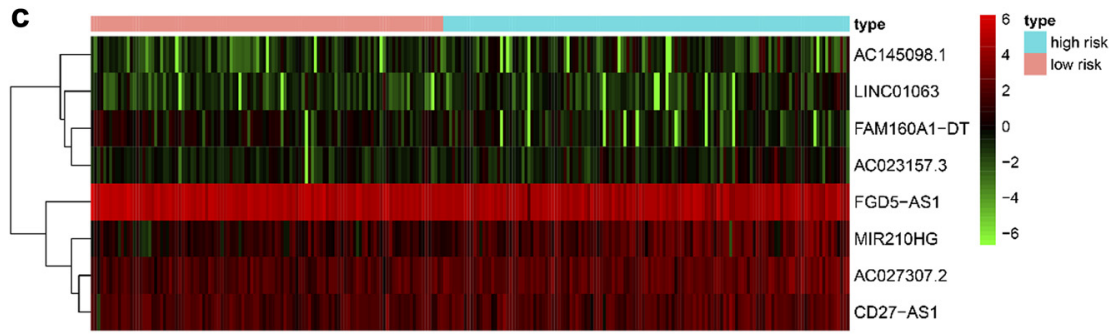

d

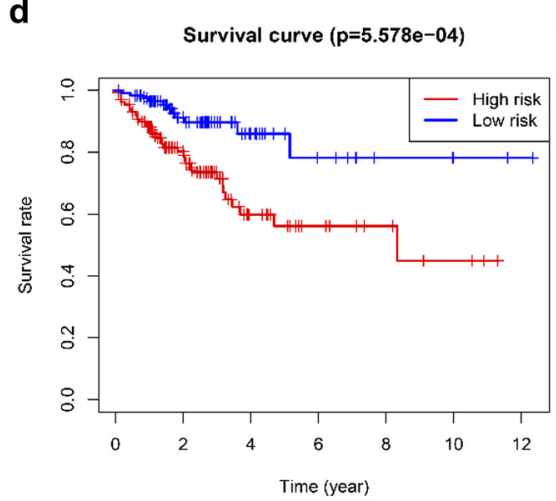

e

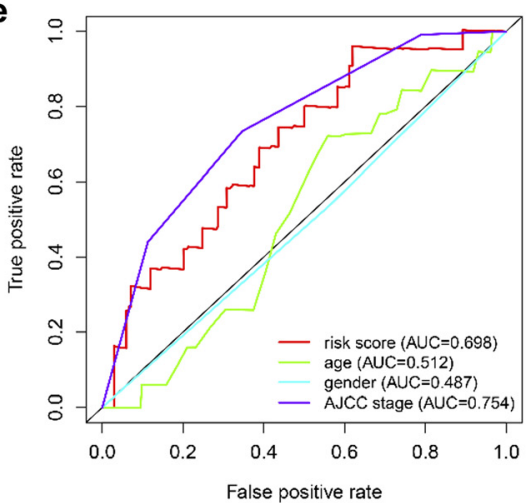

Fig. 2. 
C

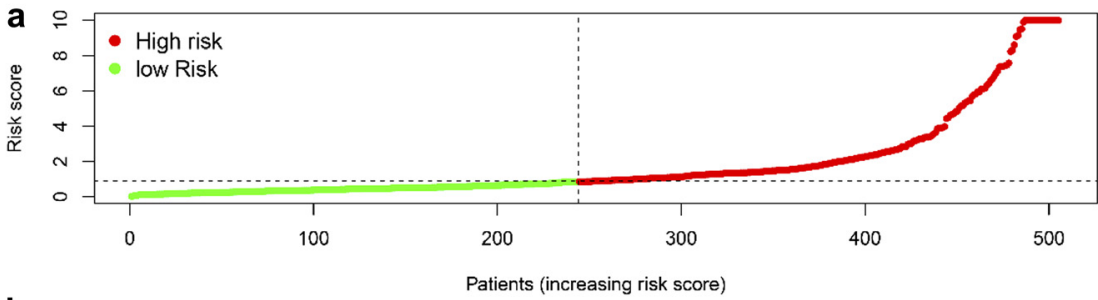

b

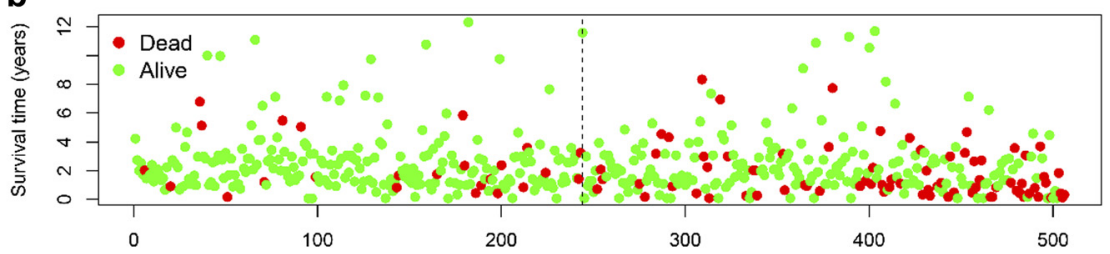

Patients (increasing risk score)

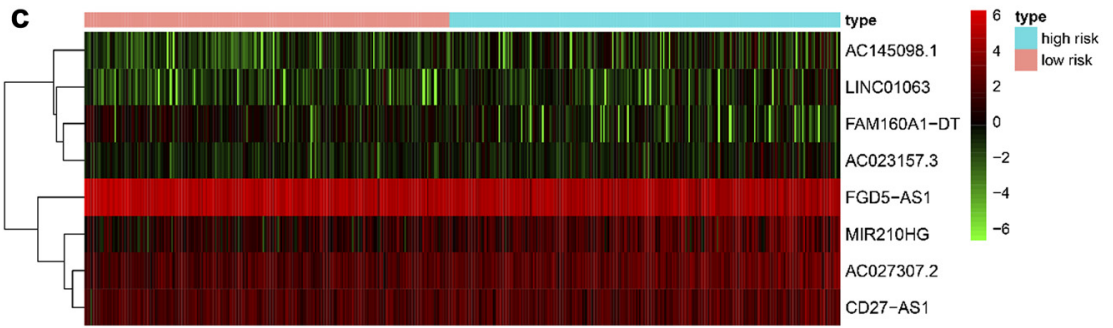

d

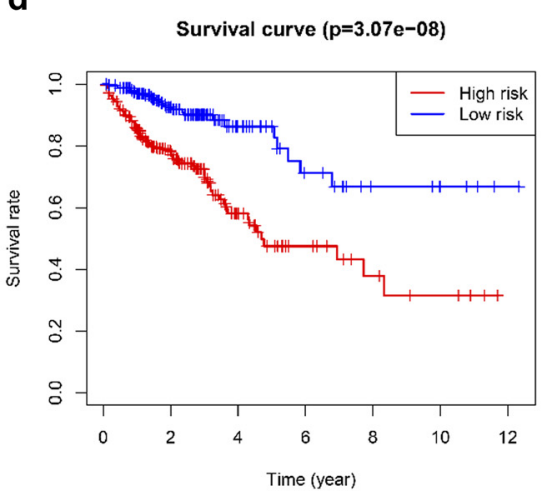

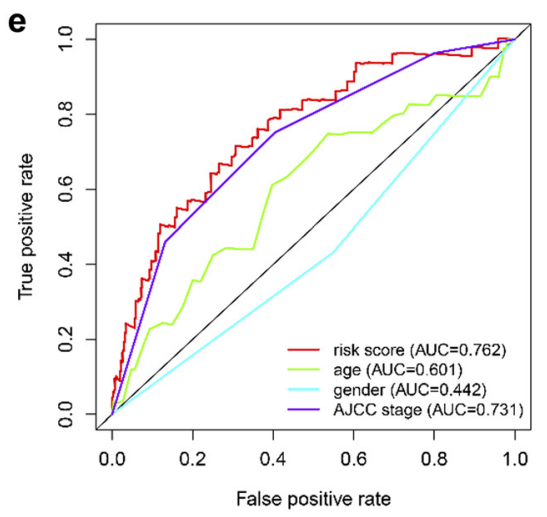

Fig. 2. 

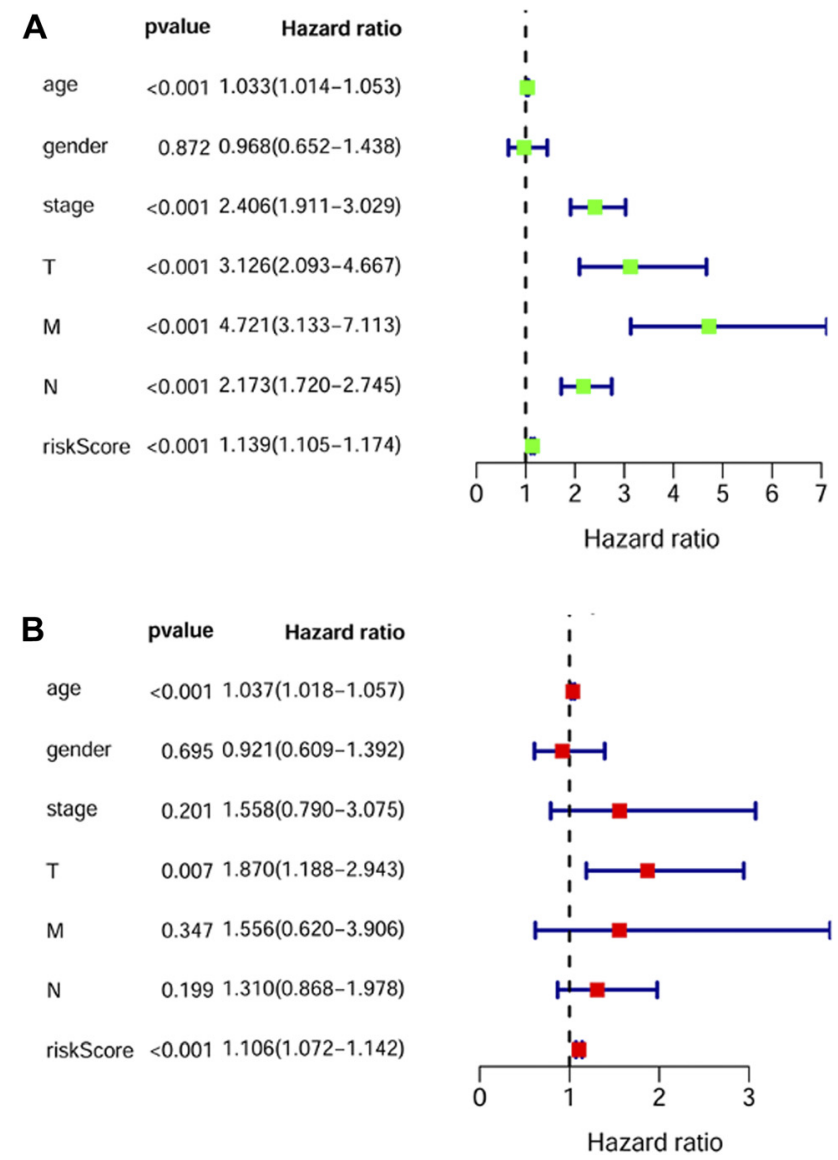

Fig. 3. Identifying the independent prognostic parameters in the whole dataset.

(A) The prognostic effect analyses of the autophagy-related lncRNAs signature and commonly used prognostic factors using univariate Cox regression model. (B) The independent prognostic effect analyses of the autophagy-related lncRNAs signature and commonly used prognostic factors using multivariate Cox regression model. lncRNAs: long non-coding RNAs

stage, and risk score calculated from the eight-lncRNA signature were independent prognostic factors for overall survival.

\section{Clinical value of the IncRNA signature in CRC patients}

Subsequently, we also determined the clinical value of the eight-lncRNA signature regarding age, gender, TNM stage, history of colon polyps, tumor status and so on. As presented in Table 2, the risk score was higher in distant metastasis cases, indicating that the lncRNA signature might be correlated with the migration and progression of CRC. However, no difference in risk score was observed according to gender, age, body mass index, history of colon polyps, AJCC stage or with regards to K-ras status, or mismatch repair status. 
Table 2. The clinical information of CRC patients in TCGA

\begin{tabular}{|c|c|c|c|c|c|c|}
\hline Clinicopathological Variables & & No. & $\begin{array}{l}\text { Risk score } \\
\text { Mean }\end{array}$ & SD & $\mathrm{t}$ & $P$-value \\
\hline \multirow[t]{2}{*}{ Age (year) } & $\leq 65$ & 225 & 1.731 & 2.897 & -1.948 & 0.052 \\
\hline & $>65$ & 280 & 2.338 & 4.093 & & \\
\hline \multirow[t]{2}{*}{ Gender } & Female & 233 & 2.298 & 4.21 & 1.297 & 0.195 \\
\hline & Male & 272 & 1.869 & 3.016 & & \\
\hline \multirow[t]{2}{*}{ Body mass index $\left(\mathrm{Kg} / \mathrm{M}^{2}\right)$} & $<25$ & 84 & 1.691 & 2.099 & -0.020 & 0.984 \\
\hline & $\geq 25$ & 163 & 1.697 & 2.449 & & \\
\hline \multirow{2}{*}{ History of colon polyps } & YES & 149 & 1.466 & 1.826 & -0.618 & 0.537 \\
\hline & NO & 316 & 1.361 & 1.426 & & \\
\hline \multirow[t]{2}{*}{ AJCC Stage } & I-II & 285 & 1.808 & 3.236 & -1.784 & 0.075 \\
\hline & III-IV & 220 & 2.403 & 4.045 & & \\
\hline \multirow[t]{2}{*}{ T stage } & $\mathrm{T} 1-2$ & 108 & 1.568 & 2.774 & -1.937 & 0.054 \\
\hline & T3-4 & 397 & 2.203 & 3.808 & & \\
\hline \multirow[t]{2}{*}{ M stage } & M0 & 424 & 1.812 & 2.943 & -2.385 & 0.019 \\
\hline & M1 & 81 & 3.406 & 5.877 & & \\
\hline \multirow[t]{2}{*}{$\mathrm{N}$ stage } & No & 294 & 1.788 & 3.193 & -1.971 & 0.05 \\
\hline & N1-3 & 211 & 2.456 & 4.116 & & \\
\hline \multirow[t]{2}{*}{ Tumor status } & YES & 80 & 2.92 & 5.901 & -1.976 & 0.051 \\
\hline & NO & 309 & 1.583 & 2.666 & & \\
\hline \multirow[t]{2}{*}{ Radiotherapy } & YES & 27 & 2.066 & 3.292 & -0.208 & 0.836 \\
\hline & NO & 407 & 1.928 & 3.768 & & \\
\hline \multirow[t]{2}{*}{ K-ras } & Wildtype & 27 & 1.866 & 1.906 & 0.851 & 0.399 \\
\hline & Mutation & 25 & 1.493 & 1.205 & & \\
\hline \multirow{2}{*}{$\begin{array}{l}\text { Loss of expression of mismatch repair } \\
\text { proteins }\end{array}$} & YES & 46 & 1.145 & 1.162 & 1.524 & 0.132 \\
\hline & NO & 370 & 1.437 & 1.666 & & \\
\hline
\end{tabular}

Abbreviations: TCGA: The Cancer Genome Atlas; AJCC: American Joint committee on Cancer.

\section{Gene set enrichment analysis}

To elucidate the underlying biological mechanism of the eight prognostic lncRNAs, we conducted GSEA of the two groups based on the lncRNA signature to find the enriched KEGG pathways. The results of enriched analysis for the expressed genes in the high-risk group demonstrated that they were notably enriched in tumor-related and autophagy-related pathways. A total of 47 gene sets were significantly enriched with nominal $P$ value $<0.05$, FDR $<25 \%$ and NES $>1$ in the high-risk group (Supplementary Table S3). The top 10 KEGG pathways are shown in Fig. 4A. Some of them are involved in the function of autophagy such as peroxisome and regulation of autophagy. In addition, it was noted that several pathways were involved in the development and progression of CRC, including pathways in cancer, and the TGF- $\beta$, p53, mTOR and WNT signaling pathways (Fig. 4B). The high-risk group also exhibited enrichment of one gene signature associated with colorectal cancer. Taken together, the functional enrichment analysis revealed the potential roles of the prognostic lncRNAs and autophagy in the carcinogenesis of CRC. 


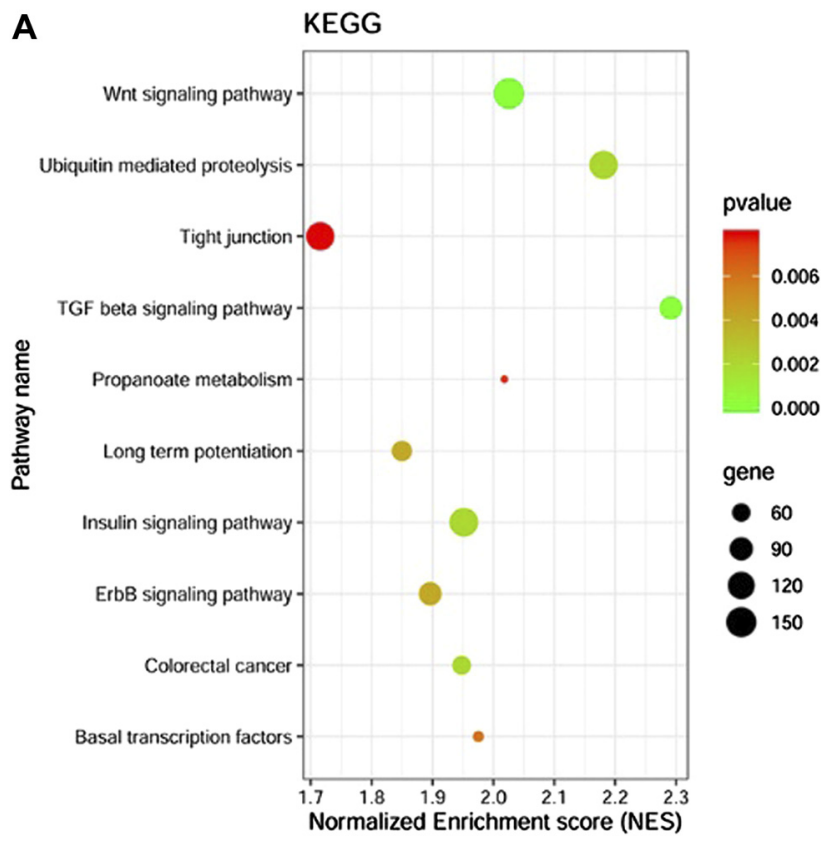

Fig. 4. Gene set enrichment analysis results based on the signature of eight autophagy-related lncRNAs. (A) Top 10 of enriched KEGG pathways in the high-risk group. (B) Gene set enrichment analysis indicated significant enrichment of KEGG cancer-related pathways in the high-risk group. lncRNAs: long noncoding RNAs; KEGG: Kyoto encyclopedia of genes and genomes; NES: normalized enrichment score 


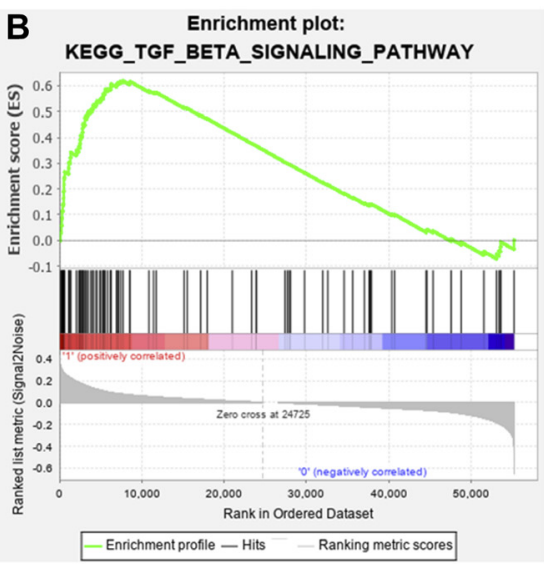

Enrichment plot: KEGG_MTOR_SIGNALING_PATHWAY

Enrichment plot: KEGG_WNT_SIGNALING_PATHWAY
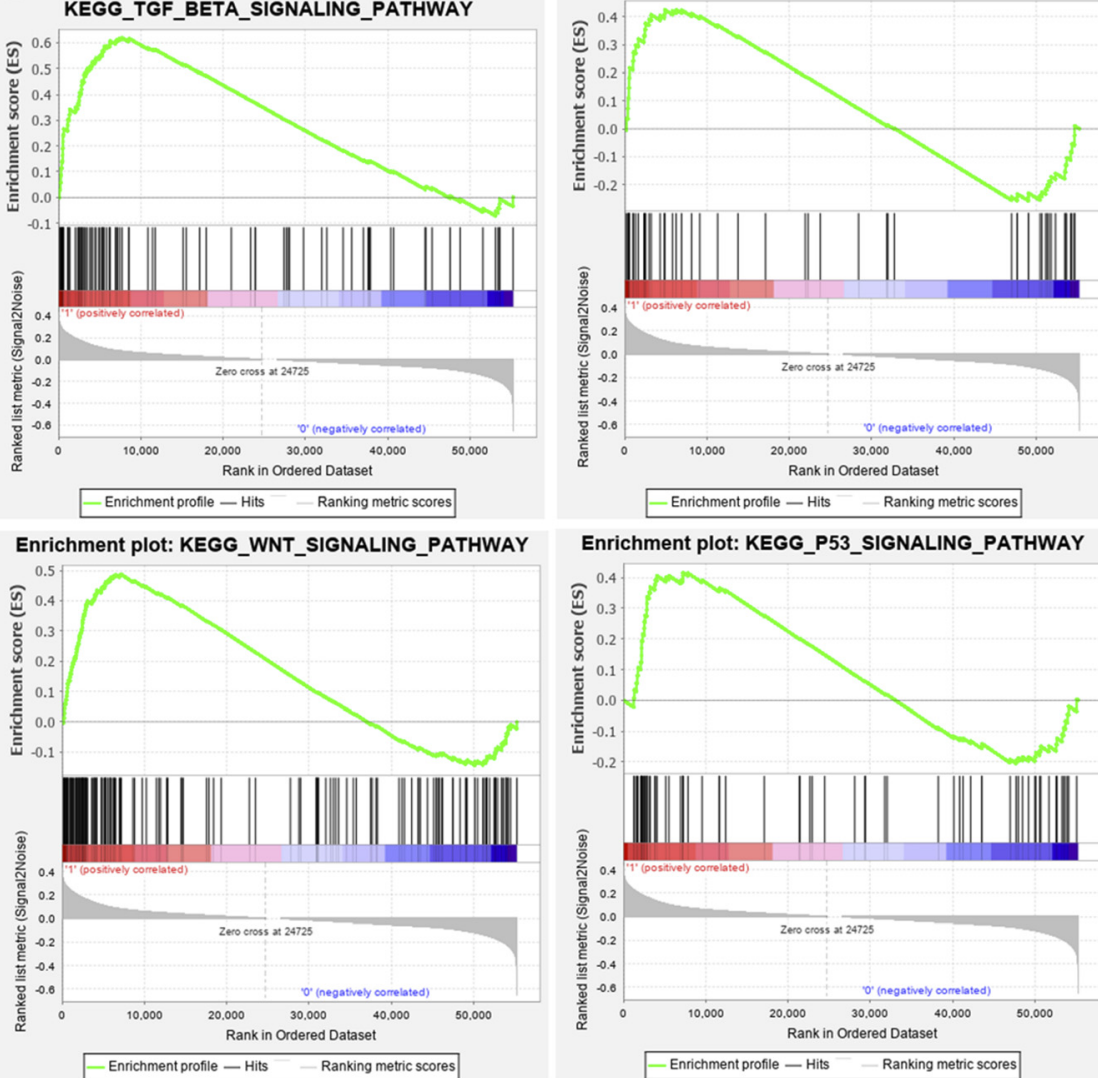

Enrichment plot: KEGG_P53_SIGNALING_PATHWAY

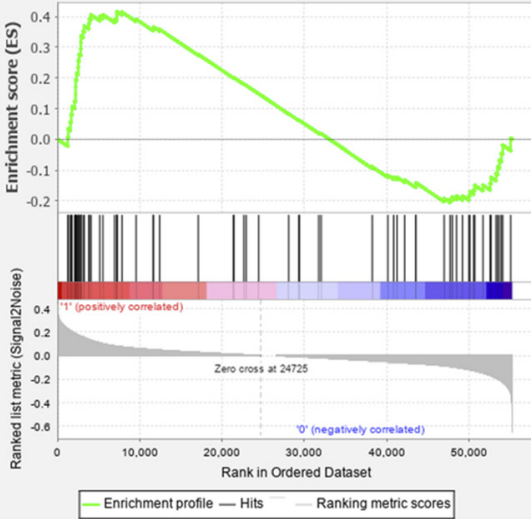

Enrichment plot: KEGG_PATHWAYS_IN_CANCER Enrichment plot: KEGG_COLORECTAL_CANCER
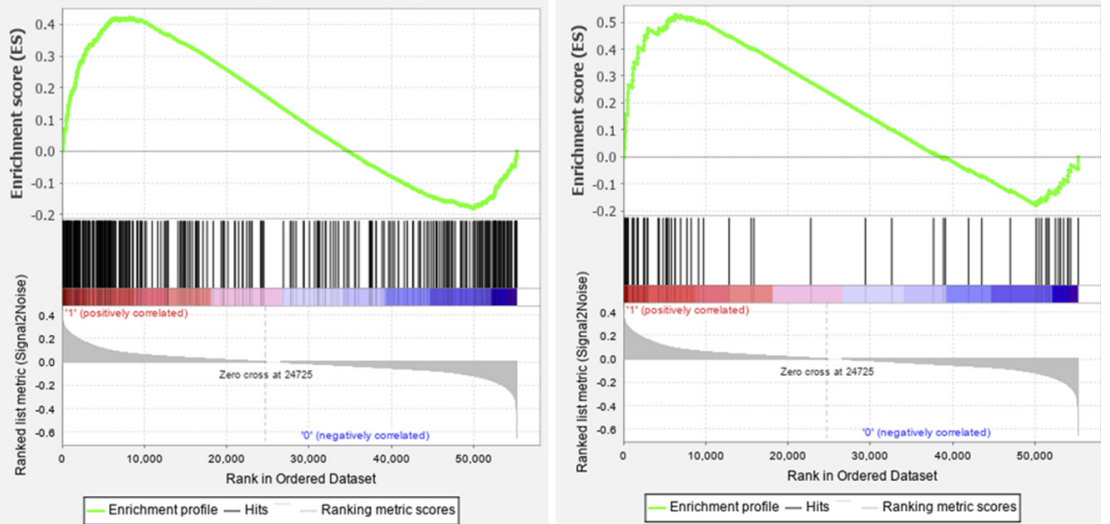

Fig. 4. 


\section{DISCUSSION}

Although extensive research efforts have been made to identify the diagnostic and prognostic indicators of CRC in the past few decades, there is still a long way to go in constructing good prognostic tools to predict the survival of CRC patients. Taking advantage of high-throughput genome sequencing technologies, a group of non-coding RNAs, IncRNAs, have been recognized and identified. An increased number of studies have shown that aberrantly expressed lncRNAs are involved in CRC progression and might be novel biomarkers for early detection, prognosis evaluation and potential targeted treatment of CRC. Moreover, sufficient evidence has shown that lncRNAs mediate post-transcriptional and transcriptional regulation of autophagy genes to modulate the complex regulatory network of autophagy [20]. Thus, the study of the prognostic value of autophagy-related lncRNAs in patients with CRC is of great strategic significance.

In the present study, to help clarify the prognostic potential of autophagy-related lncRNAs in CRC, we found 1187 lncRNAs through the coexpression network of lncRNAs and autophagyrelated genes using a large cohort from TCGA. Next, we used univariate and LASSO Cox regression analyses to identify the lncRNAs significantly correlated with the overall survival of patients with CRC and establish a prognostic eight-lncRNA signature. Further, the prognostic signature categorized CRC patients into low-risk and high-risk groups with significantly different survival outcomes, which was validated by the ROC analysis and Kaplan-Meier overall survival curve. This signature showed great consistency and performance throughout the testing set and whole set. Further univariate and multivariate Cox regression analyses indicated that the signature possessed an independent prognostic ability, indicating that it can predict the survival of CRC patients independently. In addition, we explored the underlying molecular mechanisms by using GSEA between subgroups. These results implied that the eight-lncRNA signature might serve a crucial role in the pathomechanism of CRC and could be used as a new biomarker to aid prediction of prognosis in patients with CRC.

There were eight lncRNAs in the prognostic signature and all of them had been annotated by using Ensembl database, but little is known about their biological roles in cancer progression and tumorigenesis. In our study, we found that FGD5-AS1 was an independent factor associated with good overall survival in CRC patients. FGD5-AS1 has been reported as an oncogene in non-small-cell lung cancer and oral squamous cell carcinoma, as its aberrant expression and dysregulation can promote tumorigenesis [23, 24]. Li et al. demonstrated that high-level expression of FGD5-AS1 was found in CRC cell lines, which suppressed apoptosis, accelerated proliferation and helped CRC cells to spread through negatively regulating miR-302e [25]. To date, no study has focused on the expression of FGD5-AS1 in the CRC tissue. The paradoxical results of our study may be due to the type of experiment used in the previous study, therefore further clinical trials are warranted to delineate the role of FGD5-AS1 in patients suffering from CRC. MIR210HG was found to have a positive correlation with risk score (regression coefficient $=0.140$ ), which demonstrated that higher MIR210HG expression was associated with poorer CRC prognosis. Consistent with our studies, MIR210HG has been found to be overexpressed in lung cancer [26], osteosarcoma [27], invasive breast cancer [28], and hepatocellular carcinoma [29], and its overexpression predicts a poor prognosis and a lower overall survival rate. A recent study revealed that MIR210HG may exert its effects in colon cancer through the modulation of energy metabolism and cell adhesion, but experimental evidence should be provided in future studies [30]. CD27-AS1 has been reported to be downregulated in cervical carcinoma [31]. 
However, studies on CD27-AS1 in cancer are, so far, limited in number. In addition, there weren't any reports on the other five lncRNAs according to a PubMed search. Therefore, further investigations are needed to clarify the functions of these lncRNAs in CRC and other cancers.

In recent years, remarkable advances in genomic techniques have enabled rapid identification of an increasing number of genomic alterations involved in the occurrence, development and progression of CRC, such as common mutations, chromosomal changes and translocations [32]. Multiple genomic aberrations have been reported to influence critical functions within the cell (p53 and cell-cycle regulation) and vital pathways (TGF- $\beta$, WNT and MAPK/PI3K) [33]. In the present study, KEGG pathway analysis indicated that the functions of the eight lncRNAs were potentially correlated with the TGF- $\beta$, p53, mTOR and WNT signaling pathways. These results reveal that the eight-lncRNA signature contributes to important cancer pathways, which may add an important piece of evidence for targeted therapy for CRC. Notably, these signaling pathways are known to regulate autophagy. Among these, mTOR signaling is an important signaling pathway that acts as a central regulator in the complex process of autophagy and promotes cancer progression, and mTOR inhibitors have provided therapeutic efficacy toward CRC [34]. Besides, there is already evidence that p53, a key tumor suppressor gene, can either stimulate or inhibit autophagy. For example, p53 can directly activate the transcription of a set of autophagy-relate genes including Atg7 and Ulk1 [35]. In contrast with this study, p53 has been identified to prevent the activation of autophagy in several cell lines in response to several distinct stimuli [36]. The two pathways of p53 and autophagy are now functionally linked, providing new insights into their mechanisms in cancer progression and tumorigenesis. Understanding the functional interactions between the autophagy process and the p53 pathway may have some benefit for the treatment of CRC.

To the best of our knowledge, the eight autophagy-related lncRNA signature has not been previously reported, and showed improved performance compared to the conventional clinical parameters such as TNM stage, implying a more precise reflection of the substantial heterogeneity of CRC. It is anticipated that the signature could be a valuable and useful biological tool for CRC diagnosis and prognosis. However, this study has limitations. First, all of our samples and clinical data were based on the TCGA dataset. The prognostic value of the eight-lncRNA signature should be further validated in cohorts with larger sample sizes from other datasets in the future. Furthermore, the information on several other important clinicopathological features, such as adjuvant chemotherapy use, was unavailable in these datasets. Third, the expression of the eight lncRNAs warrants further investigation in clinical trials. Fourth, based on data obtained from TCGA, we performed a bioinformatics analysis to identify the prognostic autophagy-related lncRNAs, and the functions of the lncRNAs were not characterized using in vitro or in vivo experimentation. Further studies are required to delineate the role of these lncRNAs in CRC, measure their effects on cell autophagy and determine the mechanisms involved in the progression and tumorigenesis of CRC.

Taken together, we identified a signature of eight autophagy-related lncRNAs for predicting overall survival in CRC patients by analyzing a coexpression network of lncRNAs and autophagyrelated genes. It accurately separates CRC patients with unfavorable prognosis from those with favorable prognosis, which may assist physicians in formulating more efficient therapeutic strategies. The present study not only shows the value of an eight autophagy-related lncRNAs signature as a promising classification tool for CRC diagnosis and prognosis but also potentially opens a new field in the role and regulation of autophagy in the tumorigenesis and progression of CRC. 
Conflict of interest: The authors declare no conflict of interest.

Author contributions: DYZ conceived and designed the analysis and wrote the manuscript. XZS took part in analyzing the data and writing the manuscript. SDL analyzed the data and interpreted the results. SKY designed the study and revised the manuscript. All authors read and approved the final manuscript.

\section{ACKNOWLEDGMENTS}

The authors thank the TCGA project for providing invaluable datasets for statistical analyses. This work was supported by the National Key Development Plan for Precision Medicine Research (2017YFC0910002).

\section{SUPPLEMENTARY MATERIAL}

Supplementary data to this article can be found online at https://doi.org/10.1556/2060.2021. 00125.

\section{REFERENCES}

1. Siegel RL, Miller KD, Jemal A. Cancer statistics, 2017. CA Cancer J Clin 2017; 67: 7-30.

2. Arnold M, Sierra MS, Laversanne M, Soerjomataram I, Jemal A, Bray F. Global patterns and trends in colorectal cancer incidence and mortality. Gut 2017; 66: 683-91.

3. Li Y, He M, Zhou Y, Yang C, Wei S, Bian X, et al. The prognostic and clinicopathological roles of PD-L1 expression in colorectal cancer: a systematic review and meta-analysis. Front Pharmacol 2019; 10: 139 .

4. Markle B, May EJ, Majumdar AP. Do nutraceutics play a role in the prevention and treatment of colorectal cancer? Cancer Metastasis Rev 2010; 29: 395-404.

5. Weiser MR, Gönen M, Chou JF, Kattan MW, Schrag D. Predicting survival after curative colectomy for cancer: individualizing colon cancer staging. J Clin Oncol 2011; 29: 4796-802.

6. Guinney J, Dienstmann R, Wang X, de Reynifîs A, Schlicker A, Soneson C, et al. The consensus molecular subtypes of colorectal cancer. Nat Med 2015; 21: 1350-6.

7. Johansson I, Nilsson C, Berglund P, Lauss M, Ringnér M, Olsson H, et al. Gene expression profiling of primary male breast cancers reveals two unique subgroups and identifies $\mathrm{N}$-acetyltransferase-1 (NAT1) as a novel prognostic biomarker. Breast Cancer Res 2012; 14: R31.

8. Fîlfan M, Sandu RE, Zăvăleanu AD, Gresita A, Glavan DG, Olaru DG, et al. Autophagy in aging and disease. Rom J Morphol Embryol 2017; 58: 27-31.

9. Saxton RA, Sabatini DM. mTOR signaling in growth, metabolism, and disease. Cell 2017; 168: 960-76.

10. Levine B, Kroemer G. Autophagy in the pathogenesis of disease. Cell 2008; 132: 27-42.

11. Devenport SN, Shah YM. Functions and implications of autophagy in colon cancer. Cells 2019; 8. 
12. Niklaus M, Adams O, Berezowska S, Zlobec I, Graber F, Slotta-Huspenina J, et al. Expression analysis of LC3B and p62 indicates intact activated autophagy is associated with an unfavorable prognosis in colon cancer. Oncotarget 2017; 8: 54604-15.

13. Liu PF, Leung CM, Chang YH, Cheng JS, Chen JJ, Weng CJ, et al. ATG4B promotes colorectal cancer growth independent of autophagic flux. Autophagy 2014; 10: 1454-65.

14. Janku F, McConkey DJ, Hong DS, Kurzrock R. Autophagy as a target for anticancer therapy. Nat Rev Clin Oncol 2011; 8: 528-39.

15. Amaravadi RK, Lippincott-Schwartz J, Yin XM, Weiss WA, Takebe N, Timmer W, et al. Principles and current strategies for targeting autophagy for cancer treatment. Clin Cancer Res 2011; 17: 654-66.

16. Sui X, Chen R, Wang Z, Huang Z, Kong N, Zhang M, et al. Autophagy and chemotherapy resistance: a promising therapeutic target for cancer treatment. Cell Death Dis 2013; 4: e838.

17. Newton PT. New insights into niclosamide action: autophagy activation in colorectal cancer. Biochem J 2019; 476: 779-81.

18. Morris KV, Mattick JS. The rise of regulatory RNA. Nat Rev Genet 2014; 15: 423-37.

19. Mowers EE, Sharifi MN, Macleod KF. Autophagy in cancer metastasis. Oncogene 2017; 36: 1619-30.

20. Zhang J, Wang P, Wan L, Xu S, Pang D. The emergence of noncoding RNAs as Heracles in autophagy. Autophagy 2017; 13: 1004-24.

21. Zheng Y, Tan K, Huang H. Long noncoding RNA HAGLROS regulates apoptosis and autophagy in colorectal cancer cells via sponging miR-100 to target ATG5 expression. J Cell Biochem 2019; 120: 3922-33.

22. Subramanian A, Tamayo P, Mootha VK, Mukherjee S, Ebert BL, Gillette MA, et al. Gene set enrichment analysis: a knowledge-based approach for interpreting genome-wide expression profiles. Proc Natl Acad Sci U S A 2005; 102: 15545-50.

23. Fan Y, Li H, Yu Z, Dong W, Cui X, Ma J, et al. Long non-coding RNA FGD5-AS1 promotes non-small cell lung cancer cell proliferation through sponging hsa-miR-107 to up-regulate FGFRL1. Biosci Rep $2020 ; 40$.

24. Liu L, Zhan Y, Huang Y, Huang L. LncRNA FGD5-AS1 can be predicted as therapeutic target in oral cancer. J Oral Pathol Med 2020; 49: 243-52.

25. Li D, Jiang X, Zhang X, Cao G, Wang D, Chen Z. Long noncoding RNA FGD5-AS1 promotes colorectal cancer cell proliferation, migration, and invasion through upregulating CDCA7 via sponging miR-302e. In Vitro Cell Dev Biol Anim 2019; 55: 577-85.

26. Yu H, Xu Q, Liu F, Ye X, Wang J, Meng X. Identification and validation of long noncoding RNA biomarkers in human non-small-cell lung carcinomas. J Thorac Oncol 2015; 10: 645-54.

27. Li J, Wu QM, Wang XQ, Zhang CQ. Long noncoding RNA miR210HG sponges miR-503 to facilitate osteosarcoma cell invasion and metastasis. DNA Cell Biol 2017; 36: 1117-25.

28. Li XY, Zhou LY, Luo H, Zhu Q, Zuo L, Liu GY, et al. The long noncoding RNA MIR210HG promotes tumor metastasis by acting as a ceRNA of miR-1226-3p to regulate mucin-1c expression in invasive breast cancer. Aging (Albany NY) 2019; 11: 5646-65.

29. Wang Y, Li W, Chen X, Li Y, Wen P, Xu F. MIR210HG predicts poor prognosis and functions as an oncogenic lncRNA in hepatocellular carcinoma. Biomed Pharmacother 2019; 111: 1297-301.

30. Ruan Z, Xu Z, Li Z, Lv Y. Integral analyses of survival-related long non-coding RNA MIR210HG and its prognostic role in colon cancer. Oncol Lett 2019; 18: 1107-16.

31. Roychowdhury A, Samadder S, Das P, Mazumder DI, Chatterjee A, Addya S, et al. Deregulation of H19 is associated with cervical carcinoma. Genomics 2020; 112: 961-70.

32. Mármol I, Sánchez-de-Diego C, Pradilla Dieste A, Cerrada E, Rodriguez Yoldi MJ. Colorectal carcinoma: a general overview and future perspectives in colorectal cancer. Int J Mol Sci 2017; 18.

33. Comprehensive molecular characterization of human colon and rectal cancer. Nature 2012; 487: 330-7. 
34. Zhu Z, Yang C, Iyaswamy A, Krishnamoorthi S, Sreenivasmurthy SG, Liu J, et al. Balancing mTOR signaling and autophagy in the treatment of Parkinson's disease. Int J Mol Sci 2019; 20.

35. Tasdemir E, Maiuri MC, Galluzzi L, Vitale I, Djavaheri-Mergny M, D'amelio M, et al. Regulation of autophagy by cytoplasmic p53. Nat Cell Biol 2008; 10(6): 676-87. Available from: https://doi.org/10.1038/ ncb1730. 18454141.

36. Broz DK, Mello SS, Bieging KT, Jiang D, Dusek RL, Brady CA, et al. Global genomic profiling reveals an extensive p53-regulated autophagy program contributing to key p53 responses. Genes Dev 2013; 27(9): 1016-31. Available from: https://doi.org/10.1101/gad.212282.112. 23651856. 